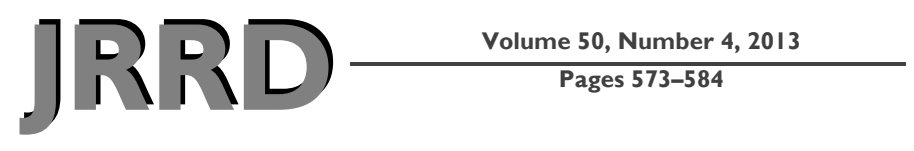

\title{
Evaluating psychoacoustic measures for establishing presence of tinnitus
}

\author{
James A. Henry, PhD; ${ }^{1-2 *}$ Garnett P. McMillan, PhD; ${ }^{1}$ Emily J. Thielman, MS; ${ }^{1}$ Gino Galvez, PhD; ${ }^{1}$ Tara L. $^{2}$ \\ Zaugg, AuD; ${ }^{1}$ Edward Porsov, MS; ${ }^{2}$ Grayson Silaski, BSEE ${ }^{1}$ \\ ${ }^{1}$ Department of Veterans Affairs (VA) Rehabilitation Research and Development National Center for Rehabilitative \\ Auditory Research, VA Medical Center, Portland, OR; ${ }^{2}$ Department of Otolaryngology/Head and Neck Surgery, Ore- \\ gon Health \& Science University, Portland, OR
}

\begin{abstract}
The Department of Veterans Affairs (VA) considers tinnitus a disability. Veterans can claim tinnitus as a "serviceconnected" disability if the tinnitus is thought to be connected to military service. The VA adjudicates each claim and determines whether reasonable evidence exists to support it. Currently, determining the presence of tinnitus is based on subjective reporting - objective measures do not exist. The aim of this study was to develop and document a test for detecting the presence/absence of tinnitus with high confidence. Using our computer-automated, self-guided tinnitus evaluation system, we conducted three phases of testing to compare psychoacoustic measures of tinnitus between participants with versus without tinnitus. Phase 1 measures included loudness match, pitch match, minimum masking level, residual inhibition, Békésy, and forced-choice double staircase. Phases 2 and 3 measures were chosen based on results of the previous phase. The number of tests and time of testing decreased during each successive phase. Differences were seen between groups; most notably, higher low-frequency loudness matches and higher median pitch matches were observed for participants with tinnitus. Results of this study suggest that further efforts can produce a defined psychoacoustic test battery for identifying the presence/absence of tinnitus.
\end{abstract}

Key words: compensation, hearing disorders, loudness matching, loudness perception, malingering, pitch matching, pitch perception, rehabilitation, reliability of results, tinnitus, tinnitus diagnosis.

\section{INTRODUCTION}

Chronic tinnitus is the persistent perception of sound that has no external source. Numerous studies indicate that tinnitus is experienced by $10-15$ percent of adults [1]. An estimated 3-4 million U.S. military Veterans have tinnitus, of whom up to 1 million require clinical intervention [2]. It was recently reported that Veterans have twice the prevalence of tinnitus as non-Veterans [3]. The greater prevalence of tinnitus for Veterans compared with non-Veterans has also been reported by Adams et al. [4]. It would seem likely that noise exposure in the military

Abbreviations: ANSI = American National Standards Institute, AUC = area under Receiver Operating Characteristic curve, $\mathrm{dB}$ HL = decibels hearing level, $\mathrm{dB}$ SL = decibels sensation level, dB SPL = decibels sound pressure level, FCDS = forced-choice double staircase, IOM = Institute of Medicine, $\mathrm{LM}=$ loudness match, $\mathrm{MML}=$ minimum masking level, $\mathrm{PM}=$ pitch match, PTA = pure tone average, PVAMC = Portland VA Medical Center, RI = residual inhibition, $\mathrm{ROC}=$ Receiver Operating Characteristic, SBC = Schwartz Bayes Criterion, TES = Tinnitus Evaluation System, VA = Department of Veterans Affairs, VHA = Veterans Health Administration.

*Address all correspondence to James A. Henry, PhD; VA Medical Center (NCRAR), PO Box 1034, Portland, OR 97207; 503-220-8262, ext 57466; fax: 503-402-2955.

Email: james.henry@va.gov

http://dx.doi.org/10.1682/JRRD.2012.05.0090 
(weapons fire, aircraft, explosions, etc.) increases the prevalence of tinnitus in Veterans [5].

Veterans can claim tinnitus as a service-connected disability. When approved, tinnitus claims nearly always result in a 10 percent disability rating, which qualifies the claimant for monthly compensation. The numbers of tinnitus disability claims have increased dramatically in the last $10 \mathrm{yr}$, and according to the Veterans Benefits Administration, tinnitus has been the most prevalent serviceconnected disability since 2008 (www.vba.va.gov/reports/ abr/index.asp). During fiscal year 2011, 87,621 Veterans were awarded a new tinnitus service connection. By the end of fiscal year 2011, a total of 840,865 Veterans were service connected for tinnitus. Tinnitus is clearly a significant and escalating problem for Veterans and for the Department of Veterans Affairs (VA) healthcare system.

Numerous factors have increased the problem of tinnitus for the Veterans Health Administration (VHA):

1. A series of changes in Federal regulations, beginning with Public Law 104-262, the Veterans Health Care Eligibility Reform Act of 1996, and concluding with VHA Directive 2008-070 resulted in many more Veterans being eligible for VA audiology services, including the provision of hearing aids.

2. In 1999, the definition of tinnitus was changed from "persistent" tinnitus due to hearing loss, head injury, or concussion to "recurrent" (occurring repeatedly) tinnitus due to any medical condition.

3. The proliferation of books, Web sites, and organizations focusing on tinnitus has increased its visibility as a legitimate medical condition. Veterans are more aware of tinnitus and are increasingly submitting disability claims and requesting services at VA medical centers.

Patients reporting tinnitus are generally taken at their word because there would normally be no reason to dispute their complaint. Some patients, however, seek documentation to support a claim for financial compensation or to become eligible for medical services. The examiner must possess the skills to conduct such an assessment and to make qualified judgments as to the legitimacy of such claims [6]. The examiner's task is to verify whether the patient's claim is accurate and honest [7].

Performing a tinnitus assessment for litigation purposes can be a complex matter. The assessment involves several components, with each component requiring the examiner to make a decision based entirely on the claimant's subjective report. These components generally include assessing the presence, impact, etiology, and permanency of tinnitus [8].

No objective measure currently exists to prove the presence of chronic tinnitus or to verify its reported severity [5,9-10]. Assessing tinnitus is similar to assessing pain, which depends on self-report, scaling of the subjective symptom, and medical history [11]. Assessing tinnitus for litigation purposes typically involves establishing consistency between verbal responses and medical records, reliability of tinnitus psychoacoustic measures, and sufficient duration of the tinnitus to assume permanency [8]. There is further the question of functional effects (impact, distress, reactions, etc.) of the tinnitus [12]. With the VA, however, functional effects are not considered when assessing a tinnitus claim. Only two nonfunctional factors are considered when assessing a Veteran's claim of tinnitus as a service-connected disability: (1) the tinnitus must be at least recurrent (intermittent) and (2) the tinnitus must be caused by some experience while in the military (e.g., noise exposure, head trauma) [2]. Both of these factors involve the interpretation of highly subjective information derived largely, if not solely, from verbal report. VA examiners must opine as to the cause of the tinnitus and its potential connection to military service. Adjudicators rule whether the tinnitus is or is not related to military service. An affirmative ruling results in a tinnitus service-connected disability award.

We have conducted studies since 1995 to develop computer-automated, self-guided tinnitus assessment procedures that can be used in a standardized fashion [13-18]. The automated system has undergone numerous iterations and was eventually redesigned to enable clinical implementation. The Tinnitus Evaluation System (TES) now comprises a laptop computer (with custom programming) and a small patient-control box (TES Podiometer, or "Pod") that is hardwired to a set of insert earphones. Before the present study, measures obtained with the TES included (1) hearing threshold, (2) loudness match (LM), (3) pitch match (PM), (4) bandwidth match, (5) minimum masking level (MML), and (6) residual inhibition (RI). Repeated testing with research participants has documented response reliability for all these tests $[16,19-22]$. In addition, the TES has been betatested for clinical application at VA audiology clinics in Bay Pines, Florida; Biloxi, Mississippi; Portland, Oregon; San Diego, California; and Ft. Lauderdale, Florida. 
When assessing tinnitus claims, it is not possible to verify the presence of tinnitus. We have therefore conducted testing of individuals with and without subjectively reported tinnitus using the TES. Preliminary data have revealed significant differences in responses between groups [17-18]. The primary objective of the present study was to develop and document a defined test for detecting the presence/absence of tinnitus with a high degree of confidence. To accomplish this objective, we developed and evaluated an automated test battery to be administered in a single session.

\section{METHODS}

Using the TES, we conducted a series of experiments comparing responses between study participants who have tinnitus versus participants who do not have tinnitus. The study was conducted in three phases, and multiple psychoacoustic tests were conducted during each phase. Tests conducted during phase 1 included tinnitus LM, tinnitus PM, MML, RI, Békésy, and forced-choice double staircase (FCDS). The tests conducted during phases 2 and 3 were chosen based on results of the previous phase. The number of tests and the time of testing decreased during each phase.

\section{Study Participants}

For each of the three phases, two groups of participants were identified: those with self-reported chronic tinnitus ("tinnitus" group) and those reporting no tinnitus ("non-tinnitus" group). Study participants were recruited by a combination of local newspaper advertising, recruitment flyers posted at the Portland VA Medical Center (PVAMC), and by contacting individuals who had previously participated in research at the National Center for Rehabilitative Auditory Research.

Interested parties contacted the Research Coordinator, who conducted telephone screening using a standardized recruitment guide. Callers were first told, "Tinnitus is ringing, buzzing, humming, or other noises in your ears or head," and then asked, "If you listen for tinnitus in a quiet room, how often do you hear it—always, almost always, sometimes, almost never, or never?” Callers who responded "almost never" or "never" were identified as not having tinnitus. Those who responded "almost always" or "always" were identified as having tinnitus.
Those responding "sometimes" were excluded because of the intermittent nature of their tinnitus.

Candidates who passed the telephone screening were scheduled for a hearing evaluation to determine whether they were eligible for the study (described in next paragraph) and to obtain informed consent. While still on the telephone, they were informed that the study was being conducted to develop a new technique for determining whether a person has tinnitus or not. Those who did not have tinnitus were told that their task was to pretend that they experienced tinnitus and to respond accordingly with respect to all testing, questionnaires, and interview questions.

At their appointment, candidates had their hearing evaluated by an audiologist (conventional hearing threshold evaluation with results in decibels hearing level $[\mathrm{dB}$ $\mathrm{HL}]$ ). Exclusion criteria at this stage included visible signs of middle or outer ear problems, air-bone gaps of $15 \mathrm{~dB}$ at two or more frequencies in one ear, or air-bone gap of $20 \mathrm{~dB}$ or greater at any one frequency. After phase 1 , a maximum hearing loss criterion was also used; participants were excluded if they had more than one threshold of 70-75 dB HL or any one threshold of $80 \mathrm{~dB}$ HL or greater in the test ear. This maximum hearing loss criterion was imposed to ensure that participants could hear the test stimuli delivered from the TES. Qualifying candidates were consented and officially enrolled as study participants. Participants then completed basic questionnaires providing demographic data and characteristics of tinnitus (duration, tonality, location, etc.).

To balance groups with respect to degree of hearing loss, we recruited equal numbers of participants for the tinnitus and non-tinnitus groups in the following four categories: (1) younger normal to near-normal hearing, (2) younger hearing impaired, (3) older normal to nearnormal hearing, and (4) older hearing impaired. Normal to near-normal hearing was defined as all hearing thresholds within $35 \mathrm{~dB}$ HL in the test ear. "Younger" and "older" were defined as, respectively, $\leq 50$ and $>50 \mathrm{yr}$ of age for phases 1 and 2 . In phase 3 , age categories of 40 59 (younger) and 60-79 (older) yr were used. Participants received $\$ 20$ for their involvement in the study.

\section{Testing Equipment}

Audiometric testing was conducted in an Acoustic Systems (Austin, Texas) Model RE-245S double-walled sound-attenuated suite. We used the fifth-generation version of the TES, which provides completely automated 
testing sequences [13-18]. For testing with the TES, participants sat facing a computer monitor. Instruction screens on the monitor guided them through the tests. For most of the tests, a sound was presented and participants turned a dial on the Pod to control output level or frequency of the sound. Push buttons on the Pod were depressed to make response choices.

\section{Hardware}

The Pod (Figure 1) served as a user interface, enabling participants to control stimulus parameters and respond to tests. The Pod provides for signal generation and signal processing (mixing, attenuation, switching, muting, and headphone buffering). Specifications of signal parameters conform to the American National Standards Institute (ANSI) specification of audiometers (ANSI S3.6-1996) across the frequency range 125-8,000 $\mathrm{Hz}$. Controls on the Pod include a continuous rotating encoder dial that provides a single-point adjustment of signal parameters. The encoder dial is programmed to facilitate each particular test. The Pod also contains four push buttons to facilitate participant responses.

\section{Software}

The TES can test at any or all of 19 available test frequencies: 1/3-octave steps between 250 and 16,000 Hz.

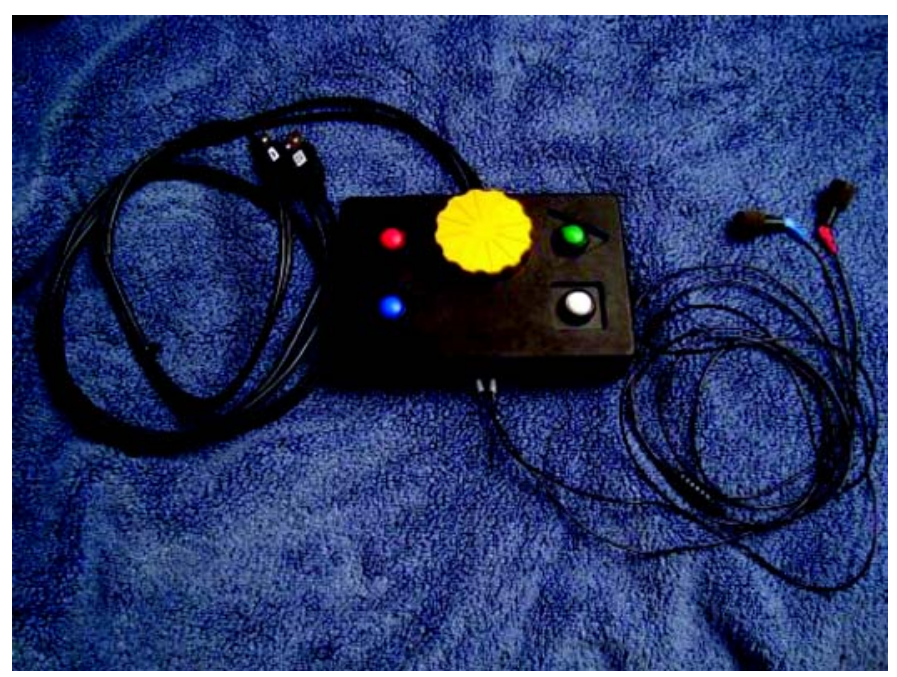

\section{Figure 1.}

Podiometer ("Pod") used by participants to respond to automated testing by turning encoder dial and depressing response buttons. Pod connects to computer and is hardwired to ER-4B insert earphones.
Tests and test parameters are preset into operator-created session templates, which enables the same battery of tests to be run on multiple patients. All test parameter options and settings are stored in a Microsoft Access database (Microsoft Corp; Redmond, Washington). Test sessions are launched by creating a new or selecting an existing patient record and choosing a preconfigured template. The template can be adjusted for an individual test subject's hearing profile in the session configuration menus. Following completion of a test session, the operator can open a report summarizing test results.

When a test session is launched, the program locks out all other functions of the computer; i.e., participants could not exit the testing program and see the setup dialogs or any other programs on the computer. Responses made with the Pod encoder dial and response buttons were recorded to the same database from which test session parameters were read.

\section{Calibration}

The Pod is a single integrated device with insert earphones ER-4B (Etymotic Research, Inc; Elk Grove Village, Illinois) permanently attached (Figure 1). The Pod was calibrated in the laboratory (using a Brüel \& Kjaer [Nærum, Denmark] type 2231A sound level meter and type 4157 ear simulator) and then delivered to the testing location. Calibration data were stored in the Pod's memory. When used for testing, the Pod was precalibrated. The Pod's calibration time stamp was checked by a "testing interface" program and compared with the one already stored in the database. If newer calibration data became available, they were downloaded from the Pod, saved in the database, and used during testing to provide calibrated stimulus levels. Previous calibration data were permanently retained in the database for tracking. A custom test template was developed to check the calibration of the Pod at regular intervals, using an Extech Instrument (Nashua, New Hampshire) Model 407768 sound level meter.

\section{Testing}

During TES testing, the audiologist was kept blinded as to whether a participant did or did not report the perception of tinnitus. The audiologist treated all participants as if they had tinnitus. 


\section{Instructions to Non-Tinnitus Participants}

Non-tinnitus participants were coached to respond to all testing as if they perceived tinnitus. They were instructed: "I want you to respond to the tests on the computer the way you would if you were trying to convince the test (or computer) that you have tinnitus." They were queried to ensure that they understood that they would be matching tones in one ear to an imagined sound (tinnitus) in the contralateral ear. Participants were not instructed to use any particular method to behave as though they were convincing the audiologist they had tinnitus. Participants determined on their own how they would respond in spite of their nonexistent tinnitus.

\section{Tinnitus Ear and Stimulus Ear}

Tinnitus matching requires individuals to distinguish between a matching tone and the tinnitus. To accomplish this, we adhere to Vernon et al.'s premise that the stimulus in one ear should be compared to the tinnitus in the contralateral ear [23-24]. Participants with tinnitus reported the ear with the more predominant tinnitus, which was designated the "tinnitus ear." The "stimulus ear" was then the contralateral ear. If the tinnitus perception was reported to be symmetrical, then participants were given the choice as to which ear received the stimulus.

For the non-tinnitus participants, if one ear was reported to have better hearing sensitivity, then that ear was designated the stimulus ear. If hearing sensitivity was symmetrical, then participants could choose which ear would be the stimulus ear. Instructions to non-tinnitus participants for performing tinnitus matching were the same as for the participants with tinnitus. The audiologist placed an insert earphone in the stimulus ear before testing.

\section{Tinnitus Loudness Matches}

Tinnitus LMs were obtained at 1/3-octave frequencies from 0.25 to $16 \mathrm{kHz}$. Obtaining an LM at a test frequency requires first determining the hearing threshold at that frequency to enable the LM to be reported in decibels sensation level (dB SL) (decibels above threshold). During testing, thresholds and LMs were recorded in decibels sound pressure level (dB SPL) (decibels referenced to $0.0002 \mu \mathrm{bar}$ ) rather than $\mathrm{dB}$ HL for two reasons: hearing threshold levels in $\mathrm{dB}$ HL have not been established for frequencies above $8 \mathrm{kHz}$ and the ER-4B insert earphones have not been documented for conventional threshold testing.
Testing started by obtaining a hearing threshold at $250 \mathrm{~Hz}$. Participants were instructed to rotate the encoder dial to the point that the pulsing test tone could "just barely be heard." When participants indicated that the instructions were understood (by pushing a response button on the Pod), the computer presented the tone at an output level selected at random from within a designated range. Participants rotated the encoder dial to find the point of minimum audibility for the tone, and then selected the threshold level by pressing the response button. A second response was obtained in the same manner. The two responses were averaged to specify the hearing threshold at that frequency.

When a threshold was obtained at $250 \mathrm{~Hz}$, an LM was then obtained at the same frequency. To ensure that participants understood the concept of loudness, an instruction screen was shown to explain the difference between pitch and loudness [21,23]. Instructions were then shown to explain the LM task. Following instructions, a continuous tone was presented at a randomized output level above the hearing threshold just established. Participants rotated the encoder dial to find the level of the tone that matched the "loudness of the tinnitus" and selected that level by pushing the response button. The tone was then again presented at a randomized output level to obtain a second LM. The two LMs were averaged to specify the LM at that frequency.

This sequence of testing (hearing threshold followed by $\mathrm{LM}$ ) was repeated at the remaining test frequencies in ascending order. Hearing thresholds and tinnitus LMs at all 19 test frequencies $(0.25-16 \mathrm{kHz}$ in $1 / 3$-octave steps) were obtained in this manner. This portion of the test would finish before 19 frequencies were tested if a participant could not hear the tone because of hearing loss, which was indicated if the participant failed to enter a response (e.g., turned the volume to maximum for more than 3 s, or "timed out," i.e., sat idle for 30 s) three times during one test run at a given frequency.

\section{Tinnitus Pitch Matches}

When LM testing was complete, on-screen instructions explained the tinnitus PM task-basically to rotate the encoder dial to find a tone that best matched the pitch of the tinnitus. The test frequencies used for pitch matching were those for which an LM was obtained. Participants rotated the encoder dial followed by a button press to identify and select the PM. This was repeated 10 times, and the computer calculated the average of the $10 \mathrm{PMs}$. 


\section{Minimum Masking Levels}

MML refers to the minimum level of broadband noise required to render a person's tinnitus inaudible. For MML testing, earphones were used in both ears. The audiologist therefore placed an insert earphone in the contralateral ("tinnitus") ear before this next stage of testing. MML testing required first determining the threshold for the broadband noise in each ear. Participants read the on-screen instructions and then proceeded to provide hearing thresholds (as for pure-tone threshold testing as just described). Following thresholds, the noise was presented bilaterally at levels $5 \mathrm{~dB}$ above the respective thresholds. Participants were instructed to choose the level of noise that "completely covers the tinnitus." They turned the encoder dial to find this level and then depressed the button selecting that level as the MML. MML testing was conducted twice, and the two responses for each ear were averaged.

\section{Residual Inhibition}

$\mathrm{RI}$ is the temporary suppression or elimination of tinnitus following auditory stimulation [25-26]. RI was tested immediately following MML testing. Participants were instructed to "listen to the sound for 1 minute" and then be prepared to report if the tinnitus sounded the same or different. The broadband noise was presented 10 $\mathrm{dB}$ above MML (in each ear respectively; if $10 \mathrm{~dB}$ above MML exceeded the output limit, then the maximum output level of $80 \mathrm{~dB}$ SPL was presented). During presentation of the noise, participants had the opportunity to reduce the level (in $5 \mathrm{~dB}$ steps) if the sound was "too loud." After $1 \mathrm{~min}$, the noise was terminated and participants were asked to indicate whether there was "any change in the tinnitus." If so, then they were asked to report the change (louder, or a percentage of the previous tinnitus loudness: $0 \%, 10 \%, 25 \%, 50 \%, 75 \%$, and $90 \%$ ).

\section{Békésy Audiometry}

The first automatic audiometer was developed by Békésy in 1947, and his technique is referred to as Békésy audiometry [27]. To perform Békésy audiometry, patients depress a button when a tone is audible and release it when the tone is inaudible. Testing can be done using either continuously variable (sweep) frequencies or discrete (fixed) frequencies. For this study, the Pod was programmed to enable Békésy audiometry through incorporation of a sweep-frequency oscillator. Software was written to perform sweep-frequency and discrete-frequency testing. For Békésy LM, participants depressed the button when the loudness of the tone was perceived to exceed the loudness of their tinnitus. They released the button when the loudness was perceived as below the loudness of their tinnitus. The tone decreased in intensity when the button was depressed and increased when the button was released. Three LM tests were performedtwo discrete-frequency tests and a sweep-frequency test. Discrete-frequency testing was done using a $1 \mathrm{kHz}$ tone and a tone at the PM frequency (determined as the average of 10 PMs recorded during the PM task). For sweepfrequency testing, a tone was swept from 1 to $8 \mathrm{kHz}$. Each Békésy test was conducted once in the continuous mode and once in the pulsed mode. Testing order (sweep vs discrete frequency and continuous vs pulsed tones) was randomized and counterbalanced.

\section{Forced-Choice Double Staircase}

The FCDS procedure is fundamentally different than tinnitus pitch matching in that patients classify a comparison stimulus relative to the tinnitus rather than matching the stimulus to the tinnitus. The laboratory version of the FCDS procedure is time consuming and requires participants to be trained in the test paradigm [28]. For our study, the FCDS protocol was simplified to the extent possible to be conducted rapidly and without training. Test instructions appeared on the computer monitor, and participants pressed the "go" button when ready to begin testing.

The procedure started with a $250 \mathrm{~Hz}$ test tone. A rough initial estimate of the tinnitus pitch was first obtained using a simple up-down staircase procedure with a large step size. It was assumed that the pitch of the test tone on the first trial was well below that of the tinnitus [29], and accordingly, the participant reported that the pitch of the test tone was below that of the tinnitus. The frequency of the test tone was then increased in octave steps until the participant reported that the pitch of the test tone was higher than that of the tinnitus. This change in response corresponded to a reversal in that the frequency of the test tone on the next trial was decreased rather than increased. The staircase procedure continued with the step size reduced to $1 / 3$ octave until the next reversal was reached. The average of the test frequencies at the two reversals provided a rough first estimate of the frequency corresponding to the tinnitus pitch.

The double staircase procedure was initiated after the test recorded three reversals. The second staircase initiated 
at one step above the previous response in the first staircase. The step size was kept at 1/3 octave for the remainder of the test to improve the precision of measurement. The two staircase procedures ran concurrently until 12 reversals were obtained for each procedure, disregarding the first 3 reversals obtained before the double staircase procedure was initiated. Each pair of reversals provided an estimate of the pitch of the tinnitus. These estimates were combined to provide a single, more precise estimate of tinnitus pitch after checking the reliability of the participant's judgments.

\section{Study Phases}

Three study phases are described in the analysis. The three phases reflect modifications to the procedures to attain more reliable, accurate, and pertinent responses from the study participants. This was an iterative study, with results of each phase determining protocol changes that were indicated for subsequent phases. Each phase involved a unique group of participants.

\section{Phase 1}

This phase included 86 participants (45 with tinnitus and 41 without). During this phase, participants completed testing while sitting alone in the sound booth. The audiologist sat in the control room and monitored the participant's activity. Testing with the TES included threshold, LM, PM, MML, RI, FCDS, and Békésy testing.

\section{Phase 2}

For phase 2, 43 new participants (23 with tinnitus and 20 without) performed testing in the sound booth. However, the audiologist sat in the booth along with the participant and was available to answer questions and provide instructions as needed for the different tests. The phase 2 version of the TES included threshold, LM, PM, MML, and FCDS testing.

\section{Phase 3}

Phase 3 involved 38 new participants (19 with tinnitus and 19 without). As dictated by the results of the phase 2 analysis, phase 3 participants only completed threshold, LM, and PM testing.

\section{Data Analysis}

The goal of the analysis was to find a set of metrics derived from the TES components that best identifies people with tinnitus. Statistical approaches used to achieve this goal are based in methods of diagnostic test development [30] and machine learning [31].

The MML in dB SL was computed from the threshold and MML responses using broadband noise; an indicator for whether RI occurred and how much RI occurred were computed from that test component. A variety of test metrics were computed from the remaining TES test component responses. Measures of central tendency and spread were computed for the PM and LM (dB SL) responses. Measures of central tendency and spread were computed from the Békésy continuous and pulse tone responses at both $1 \mathrm{kHz}$ and at the $\mathrm{PM}$ frequency. Finally, additional metrics were computed by contrasting continuous and pulse tone responses on the Békésy test component and between staircases of the FCDS response set. This collection of TES metrics comprised the predictors used in this analysis to identify tinnitus in each sample of participants.

A prediction model was developed from the phase 1 and phase 2 samples, and the phase 2 model was validated in the phase 3 sample. Simply put, the procedure randomly partitions each sample into training and test sets. The training set is used to develop a candidate statistical model that predicts the chances of having tinnitus given the TES test results. The model is then applied to the training set to determine whether the prediction model can be refined. This is repeated until an optimal model is developed from which the accuracy of the model is computed. More specifically, linear regression was used to estimate regression coefficients for each of the predictor metrics. Crossvalidated prediction accuracy, defined here as the Schwartz Bayes Criterion (SBC), was computed using random fivefold partitioning of each sample. Predictors were added or removed using stepwise regression so as to optimize the SBC statistic. This procedure was repeated until an optimal set of TES predictors was identified that best predicted tinnitus status. These predictors were then fed into a logistic regression model, which was used to compute the crossvalidated Receiver Operating Characteristic (ROC) curve and the area under the ROC curve (AUC). A technical discussion of approaches to machine learning and predictive algorithm development are given in Hastie et al. [31].

\section{RESULTS}

Table 1 summarizes the characteristics of the participants in each phase of the study. Participants without 
JRRD, Volume 50, Number 4, 2013

Table 1.

Characteristics of study participants.

\begin{tabular}{llllrrrr}
\hline Study Phase & Group & $\boldsymbol{n}$ & Age & PTA High & PTA Low & Female (\%) & Male (\%) \\
\hline 1 & No Tinnitus & 41 & 63.2 & 53.6 & 25.7 & 26.8 \\
& Tinnitus & 45 & 56.8 & 46.1 & 22.0 & 13.3 \\
2 & No Tinnitus & 20 & 54.5 & 26.7 & 12.4 & 15.0 \\
& Tinnitus & 23 & 53.4 & 34.8 & 9.2 & 4.3 & 85.7 \\
3 & No Tinnitus & 19 & 59.4 & 31.6 & 16.3 & 31.6 & 65.7 \\
& Tinnitus & 19 & 56.1 & 35.4 & 16.4 & 15.8
\end{tabular}

Note: PTA High = average of hearing thresholds at 3, 4, 6, and $8 \mathrm{kHz}$; PTA Low = average of hearing thresholds at 0.25, 0.5, 1, and $2 \mathrm{kHz}$. PTA = pure tone average.

tinnitus were on average older than participants with tinnitus across study phases. High frequency pure tone averages (PTAs) $(3,4,6$, and $8 \mathrm{kHz})$ were within $10 \mathrm{~dB}$ between groups of each study phase. A $t$-test contrasting the tinnitus and non-tinnitus subjects was not significant on high- or low-frequency PTA ( $p=0.82$ and 0.14 , respectively) and was marginally significant on age ( $p=$ 0.06). Prediction models adjusted for these effects throughout the analysis. Low-frequency PTAs (0.25, $0.50,1$, and $2 \mathrm{kHz}$ ) were all within $5 \mathrm{~dB}$ between groups of each study phase. Across study phases, men were more highly represented than women, and this discrepancy was greater for participants with tinnitus than for those without tinnitus. The greatest difference was in phase 3 , where the percentage of men in the tinnitus group was 15.8 percent higher than for men in the no tinnitus group.

\section{Phase 1 Results}

Phase 1 recruitment and testing resulted in unreliable performance on the MML test component due to low audibility of the masking sound. To avoid biasing the analysis toward better-hearing listeners, the MML and the RI, which depends on the MML result, were excluded from the model development. The final selected model included median LM (in dB SL) across all frequencies as the only necessary predictor of tinnitus. In the full phase 1 sample, the odds of having tinnitus increased by about 10 percent for each $1 \mathrm{~dB}$ increase in the median LM sensation level $(p=0.02)$. The crossvalidated ROC curve is shown in Figure 2, which gave an associated AUC of 0.65 (95\% confidence interval $=0.53-0.76)$.

During phase 1 testing, it became clear that the full battery of automated testing was imposing difficulties in concentration and accuracy on the participants. FCDS results clearly indicated poor comprehension of the testing concept, while the Békésy testing added excessive

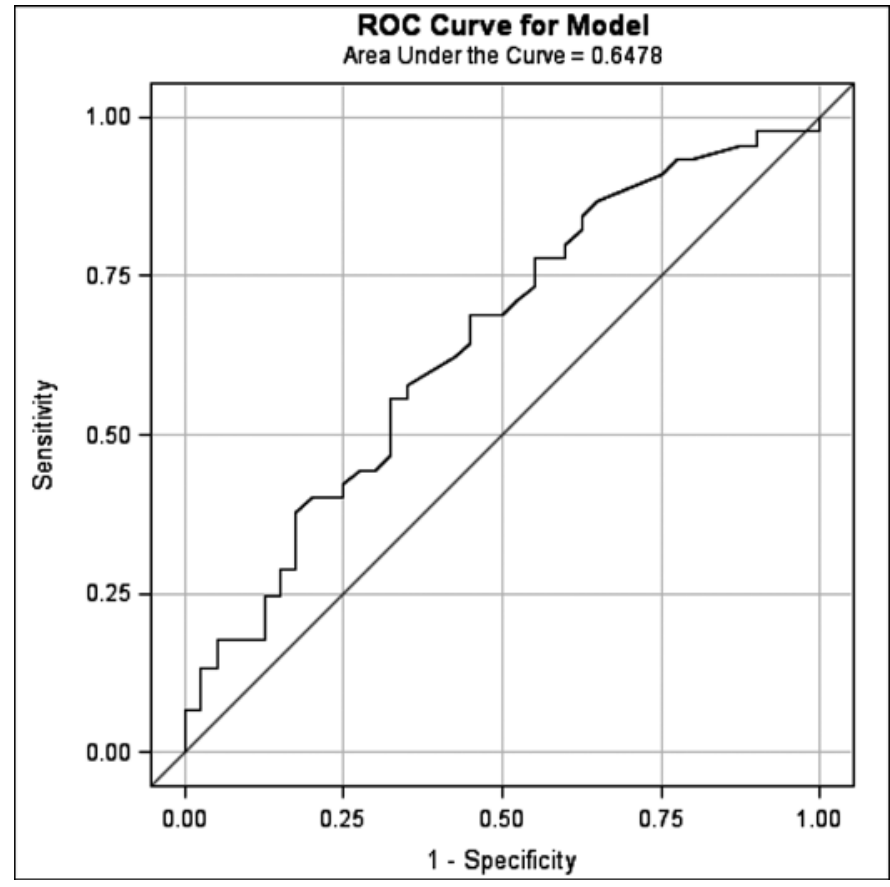

Figure 2.

Crossvalidated Receiver Operating Characteristic (ROC) for phase 1 predictive model that includes median loudness match sensation level as only predictor.

length to the test battery. Study personnel found that participants had a difficult time understanding fundamental audiologic concepts such as "pitch." As a result of these observations, in conjunction with the relatively poor accuracy of the test, the TES was revised for phase 2 of the study for testing on a subset of the initial test components and was administered with assistance from an audiologist (the audiologist sat in the sound booth with the participant and was available to answer questions and provide additional instructions). 


\section{Phase 2 Results}

For this portion of the analysis, the predictive model was modified to include age, low-frequency PTA, and high-frequency PTA in the model. We also separated the LM results into low frequency $(0.25-2 \mathrm{kHz})$ and high frequency $(2.52-8 \mathrm{kHz})$ metrics. The Békésy and RI components were removed from the test battery.

The final selected model included the mean of the low frequency LM sensation levels and the median PM, along with the age and the low- and high-frequency PTAs. Results of fitting the model to the full sample (Table 2) indicate that higher low-frequency LM sensation level $(p=0.04)$ and higher median PM $(p=0.01)$ result in higher odds of tinnitus in the sample. The crossvalidated ROC curve is shown in Figure 3, which gave an AUC of 0.88 . This indicates very good predictive accuracy in the phase 2 sample.

\section{Phase 3 Results}

The regression coefficients in Table 2 allow one to compute a TES score for the new sets of responses gathered in the phase 2 sample. These scores are computed as-

TESscore $=-7.1227+$ Age $\cdot 0.00831+$ PTAlow $\cdot$ $-0.0553+$ PTAhigh $\cdot 0.0998+$ LMSLMeanLow $\cdot 0.1210$ + PMMed $\cdot 0.000614$.

Predictive accuracy of the fitted model is shown in Figure 4. The AUC was 0.68 (95\% confidence interval = $0.50-0.86$ ), which is considerably less than the phase 2 result.

\section{DISCUSSION}

Before conducting this study, we hypothesized that performing a battery of tinnitus psychoacoustic tests in a

Table 2.

Logistic regression coefficients for the final model fit to the full phase 2 sample.

\begin{tabular}{lcc}
\hline Parameter & Estimate & $\boldsymbol{p}$-Value \\
\hline Intercept & -7.1227 & 0.07 \\
Age & 0.00831 & 0.90 \\
PTA Low & -0.0553 & 0.42 \\
PTA High & 0.0998 & 0.03 \\
Low Frequency Loudness & 0.1210 & 0.04 \\
$\quad$ & \\
$\quad$ Match Sensation Level & 0.000614 & 0.01 \\
Median Pitch Match & Note: PTA High = average of hearing thresholds at 3, 4, 6, and 8 kHz; PTA \\
Low = average of hearing thresholds at 0.25, 0.5, 1, and 2 kHz. \\
PTA = pure tone average. \\
\hline \hline
\end{tabular}

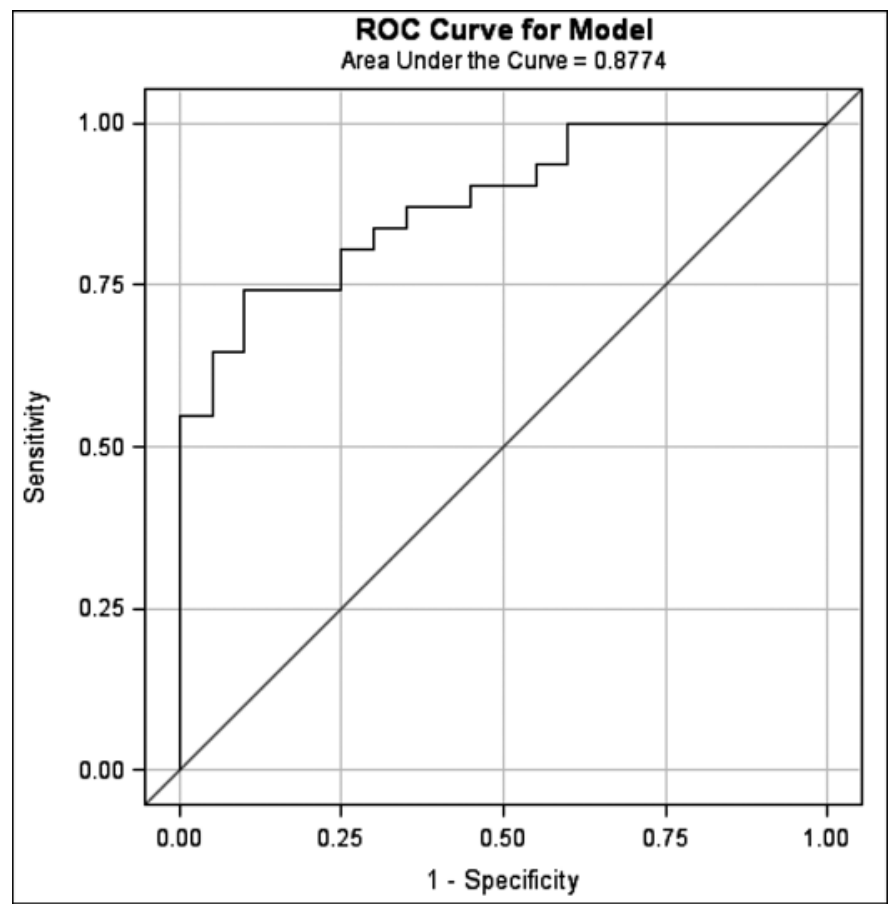

Figure 3.

Crossvalidated Receiver Operating Characteristic (ROC) for phase 2 predictive algorithm that includes age, low-frequency pure tone average (PTA), high-frequency PTA, low-frequency loudness match sensation level, and median pitch match as optimal predictors.

standardized manner would enable the identification of the presence of tinnitus with a high degree of accuracy. The initial test battery, as used during phase 1, included testing for thresholds, LM, PM, MML, RI, as well as performing Békésy and FCDS testing. All these tests were conducted automatically by the TES, and participants performed testing while sitting alone in a sound booth (with an audiologist available in the adjoining control room). In phase 1 , we discovered that most participants had difficulty with the length and complexity of testing. It became apparent that it was necessary to keep the testing protocol as short and simple as possible.

For phase 2, we simplified the test protocol by eliminating testing for RI and Békésy, because these tests did not enhance the accuracy of tinnitus prediction and were the most time consuming. In addition, the audiologist sat in the sound booth with the participant to explain the different tests and to respond immediately to questions. Testing for phase 2 included hearing thresholds, LM, PM, MML, and FCDS. Comparison of the tinnitus and 


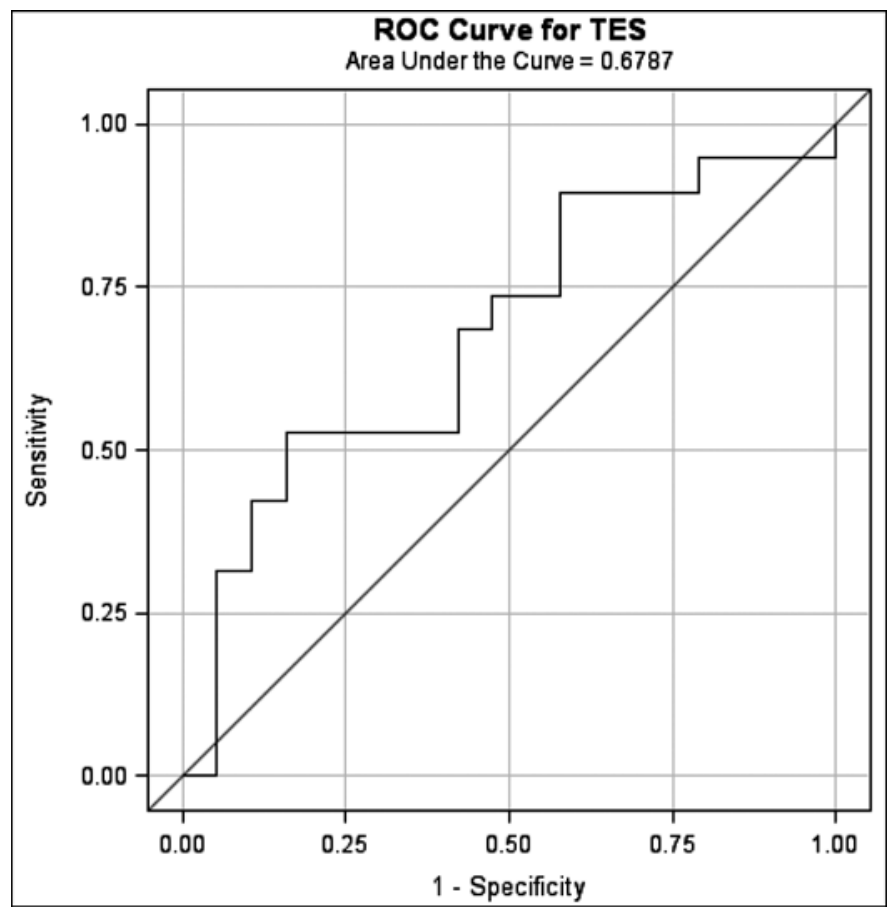

Figure 4.

Receiver Operating Characteristic (ROC) for phase 3 validation using predictive model developed in phase 2. TES = Tinnitus Evaluation System.

non-tinnitus groups revealed that the tinnitus group showed significantly higher mean LM (dB SL) in the low frequency range and significantly higher median PM. Phase 3 was then conducted to confirm the findings of phase 2.

Before phase 3, the test battery was reduced to include only those measures that appeared to more accurately differentiate between the study groups. For this reason, MML and FCDS testing were eliminated. MML testing was omitted because in phases 1 and 2, participants with tinnitus responded very similarly to participants without tinnitus. FCDS testing had proven difficult for participants to comprehend despite the availability of further instruction from the audiologist added during phase 2, and it was making the testing task more difficult without bearing any significant differences between the tinnitus and non-tinnitus groups. Except for the omission of MML and FCDS testing, phase 3 was conducted in an identical manner as phase 2 . As expected, the differences seen for phase 2 were not as pronounced for phase 3, although significant differences were again seen for lowfrequency mean LM and median PM.
Results of audiologic testing are often thought to provide the most valid evidence to support tinnitus claims $[8,32]$. Although there are documented tests for detecting exaggerated hearing loss [33-34], no such test exists to assess patients suspected of feigning tinnitus. Analysis of data from our current study suggests that results from more than one tinnitus psychoacoustic test should be evaluated in aggregate to optimize the accuracy of determining whether or not a person has tinnitus as claimed. The response "profile" should be evaluated in relation to other relevant factors. Dobie has proposed that these additional factors should include an "unsolicited" complaint of tinnitus, plausible and consistent history, patient credibility, early documentation of tinnitus, and unexaggerated hearing loss [8].

VA clinicians and benefits specialists are tasked with determining whether Veterans' auditory problems are the result of prior military service versus other causes. This determination can be problematic given that it is oftentimes made years after the Veterans' military discharge. To better understand cause-and-effect, a congressionally mandated Institute of Medicine (IOM) landmark report called "Noise and military service: Implications for hearing loss and tinnitus" recommended that researchers "[e]stablish cohorts of military veterans with various documented noise exposures, immediately upon discharge, and survey them periodically for ototoxic exposures, subsequent non-military noise exposures, and hearing function, as well as presence and severity of tinnitus, in order to determine whether there is a delay in the effects of military noise exposure. These cohorts will need to be followed through the remainder of members' lifetimes, but this longitudinal study will reveal elements of the natural history of noise-induced hearing loss and tinnitus that otherwise will not be determined." [35, p. 208]. Fulfillment of this IOM recommendation will require conducting a longitudinal cohort study in which data are gathered at incremental time points over Veterans' lifetimes, allowing an examination of the effects and interplay between military and postmilitary exposures and delayed-onset audiologic problems.

\section{CONCLUSIONS}

A major challenge of tinnitus is that the subjective nature of the condition makes it very difficult to know whether or not the condition actually exists as claimed. 
We have conducted computer-automated testing of individuals with and without tinnitus, with the goal of developing methodology to objectively detect the presence of tinnitus. This study has not resulted in a defined test for this purpose, although the data reveal significant differences in responses between groups with versus without tinnitus. Further work is still needed to develop and document a defined test for detecting the presence/absence of tinnitus with a high degree of confidence.

\section{ACKNOWLEDGMENTS}

\section{Author Contributions:}

Study concept and design: J. A. Henry, G. P. McMillan, E. J. Thielman, T. L. Zaugg.

Acquisition of data: E. J. Thielman, G. Galvez, T. L. Zaugg.

Drafting of manuscript: J. A. Henry, G. P. McMillan.

Critical revision of manuscript for important intellectual content:

J. A. Henry, G. P. McMillan, E. J. Thielman, T. L. Zaugg, E. Porsov, G. Silaski.

Statistical analysis: G. P. McMillan, E. J. Thielman.

Obtained funding: J. A. Henry.

Study supervision: J. A. Henry.

Administrative support: E. J. Thielman.

Technical support: E. Porsov, G. Silaski.

Financial Disclosures: VA and Oregon Health \& Science University own a technology used in this research. Sonitus Medical (San Mateo, California) has licensed the technology and may have an interest in the results of this study. Mr. Silaski, Mr. Porsov, VA, and Oregon Health \& Science University have rights to royalties from the licensing agreement with Sonitus Medical.

Funding/Support: This material was based on work supported by the VHA Rehabilitation Research and Development Service (grants C4698R and F7070S).

Additional Contributions: We are grateful to Harry Levitt, $\mathrm{PhD}$, and Jim Steiger, $\mathrm{PhD}$, for their valuable consultation services to develop the FCDS and Békésy tests, respectively. Special thanks to Pat Feeney, PhD; Christine Kaelin, MBA; John McDermott, PhD; and Sara Ruth O’Dell, AuD, for their significant contributions to our tinnitus research. Dr. Galvez is now with the National Council of La Raza/ California State University Long Beach Center for Latino Community and Department of Psychology, California State University, Long Beach, California.

Institutional Review: This study was approved by the institutional review board committee at the PVAMC.

Participant Follow-Up: The authors do not plan to inform participants of the publication of this study.

\section{REFERENCES}

1. Hoffman HJ, Reed GW. Epidemiology of tinnitus. In: Snow JB, editor. Tinnitus: Theory and management. Lewiston (NY): BC Decker; 2004. p. 16-41.

2. Henry JA, Schechter MA, Regelein RT, Dennis KC. Veterans and tinnitus. In: Snow JB, editor. Tinnitus: Theory and management. Lewiston (NY): BC Decker; 2004. p. 337-55.

3. Folmer RL, McMillan GP, Austin DF, Henry JA. Audiometric thresholds and prevalence of tinnitus among male veterans in the United States: data from the National Health and Nutrition Examination Survey, 1999-2006. J Rehabil Res Dev. 2011;48(5):503-16. [PMID:21674401] http://dx.doi.org/10.1682/JRRD.2010.07.0138

4. Adams PF, Hendershot GE, Marano MA. Current estimates from the National Health Interview Survey, 1996. PHS 991528. Hyattsville (MD): National Center for Health Statistics; 1999.

5. Yankaskas K. Prelude: Noise-induced tinnitus and hearing loss in the military. Hear Res. 2013;295:3-8.

[PMID:22575206] http://dx.doi.org/10.1016/j.heares.2012.04.016

6. Dobie RA. Medical-legal evaluation of hearing loss, 2d ed. San Diego (CA): Singular; 2001.

7. Vernon JA. Is the claimed tinnitus real and is the claimed cause correct? In: Reich GE, Vernon JA, editors. Proceedings of the Fifth International Tinnitus Seminar; 1995 Jul; Portland, OR. Portland (OR): American Tinnitus Association; 1996. p. 395-96.

8. Dobie RA. Medico-legal aspects of tinnitus. In: Patuzzi R, editor. VIIth International Tinnitus Seminar Proceedings; 2002 Mar 5-9; Perth, Australia. 2002. p. 25-28.

9. Meikle MB, Stewart BJ, Griest SE, Henry JA. Tinnitus outcomes assessment. Trends Amplif. 2008;12(3):223-35.

[PMID:18599500]

http://dx.doi.org/10.1177/1084713808319943

10. Henry JA. Audiologic assessment of the tinnitus patient. In: Snow JB, editor. Tinnitus: Theory and management. Lewiston (NY): BC Decker; 2004. p. 220-36.

11. Turk DC, Okifuji A. Assessment of patients' reporting of pain: an integrated perspective. Lancet. 1999;353(9166): 1784-88. [PMID:10348007] http://dx.doi.org/10.1016/S0140-6736(99)01309-4

12. Meikle MB, Henry JA, Griest SE, Stewart BJ, Abrams HB, McArdle R, Myers PJ, Newman CW, Sandridge S, Turk DC, Folmer RL, Frederick EJ, House JW, Jacobson GP, Kinney SE, Martin WH, Nagler SM, Reich GE, Searchfield G, Sweetow R, Vernon JA. The Tinnitus Functional Index: development of a new clinical measure for chronic, intrusive tinnitus. Ear Hear. 2012;33(2):153-76.

[PMID:22156949]

http://dx.doi.org/10.1097/AUD.0b013e31822f67c0 
13. Henry JA, Fausti SA, Mitchell CR, Flick CL, Helt WJ. An automated technique for tinnitus evaluation. In: Reich GE, Vernon JA, editors. Proceedings of the 5th International Tinnitus Seminar; 1995 Jul. Portland, OR. Portland (OR): American Tinnitus Association; 1996. p. 325-26.

14. Henry JA, Fausti SA, Flick CL, Helt WJ, Ellingson RM. Computer-automated clinical technique for tinnitus quantification. Am J Audiol. 2000;9(1):36-49.

[PMID:10943023] http://dx.doi.org/10.1044/1059-0889(2000/002)

15. Henry JA, Flick CL, Gilbert A, Ellingson RM, Fausti SA. Comparison of manual and computer-automated procedures for tinnitus pitch-matching. J Rehabil Res Dev. 2004;41(2):121-38. [PMID:15558367] http://dx.doi.org/10.1682/JRRD.2004.02.0121

16. Henry JA, Flick CL, Gilbert AM, Ellingson RM, Fausti SA. Reliability of tinnitus loudness matches under procedural variation. J Am Acad Audiol. 1999;10(9):502-20. [PMID:10522624]

17. Henry JA, James KE, Owens K, Zaugg T, Porsov E, Silaski G. Auditory test result characteristics of subjects with and without tinnitus. J Rehabil Res Dev. 2009;46(5):619-32. [PMID:19882495] http://dx.doi.org/10.1682/JRRD.2008.11.0157

18. Henry JA, Rheinsburg B, Owens KK, Ellingson RM. New instrumentation for automated tinnitus psychoacoustic assessment. Acta Otolaryngol Suppl. 2006;126(556):34-38. [PMID:17114140] http://dx.doi.org/10.1080/03655230600895291

19. Henry JA, Flick CL, Gilbert A, Ellingson RM, Fausti SA. Reliability of hearing thresholds: computer-automated testing with ER-4B Canal Phone earphones. J Rehabil Res Dev. 2001;38(5):567-81. [PMID:11732834]

20. Henry JA, Flick CL, Gilbert A, Ellingson RM, Fausti SA. Reliability of computer-automated hearing thresholds in cochlear-impaired listeners using ER-4B Canal Phone earphones. J Rehabil Res Dev. 2003;40(3):253-64. [PMID:14582529]

21. Henry JA, Flick CL, Gilbert AM, Ellingson RM, Fausti SA. Comparison of two computer-automated procedures for tinnitus pitch matching. J Rehabil Res Dev. 2001;38(5): 557-66. [PMID:11732833]

22. Halbauer JD, Ashford JW, Zeitzer JM, Adamson MM, Lew HL, Yesavage JA. Neuropsychiatric diagnosis and management of chronic sequelae of war-related mild to moderate traumatic brain injury. J Rehabil Res Dev. 2009;46(6):757-96. [PMID:20104402]

http://dx.doi.org/10.1682/JRRD.2008.08.0119

23. Vernon J, Fenwick J. Identification of tinnitus: a plea for standardization. J Laryngol Otol. 1984;98(S9):45-53. http://dx.doi.org/10.1017/S1755146300090107

24. Vernon JA, Meikle MB. Tinnitus masking: Unresolved problems. In: Evered D, Lawrenson G, editors. Ciba Foun- dation Symposium 85 Tinnitus. London (UK): Pitman Books, Ltd; 1981. p. 239-56.

25. Vernon JA, Meikle MB. Measurement of tinnitus: An update. In: Kitahara M, editor. Tinnitus pathophysiology and management. Tokyo (Japan): Igaku-Shoin; 1988. p. 36-52.

26. Vernon J. Relief of tinnitus by masking treatment. In: English GM, editor. Otolaryngology. Philadelphia (PA): Harper \& Row; 1982. p. 1-21.

27. Békésy GV. A new audiometer. Acta Oto-Laryngol. 1947; 35:411-22. http://dx.doi.org/10.3109/00016484709123756

28. Penner MJ, Bilger RC. Consistent within-session measures of tinnitus. J Speech Hear Res. 1992;35(3):694-700. [PMID:1608262]

29. Meikle M, Walsh ET. Characteristics of tinnitus and related observations in over 1800 tinnitus patients. In: Shulman AE, Ballantyne JC, editors. Proceedings of the Second International Tinnitus Seminar, New York. Ashford (KY): Invicta Press; 1984. p. 17-21.

30. Pepe MS. The statistical evaluation of medical tests for classification and prediction. New York (NY): Oxford University Press; 2003.

31. Hastie T, Tibshirani R, Friedman J. The elements of statistical learning: Data mining, inference, and prediction. New York (NY): Springer; 2009.

32. Coles RR. Medicolegal issues. In: Tyler RS, editor. Tinnitus handbook. San Diego (CA): Singular; 2000. p. 399-417.

33. Martin FN. Pseudohypacusis. In: Katz J, editor. Handbook of clinical audiology. Baltimore (MD): Williams \& Wilkins; 1994. p. 553-67.

34. Lutolf J. Pseudohypacusis false and exaggerated hearing loss. Ear Hear. 2012;33(4):558-60. http://dx.doi.org/10.1097/AUD.0b013e3182587498

35. Humes LE, Joellenbeck LM, Durch JS, editors. Noise and military service: Implications for hearing loss and tinnitus. Washington (DC): The National Academies Press; 2006.

Submitted for publication May 15, 2012. Accepted in revised form September 14, 2012.

This article and any supplementary material should be cited as follows:

Henry JA, McMillan GP, Thielman EJ, Galvez G, Zaugg TL, Porsov E, Silaski G. Evaluating psychoacoustic measures for establishing presence of tinnitus. J Rehabil Res Dev. 2013;50(4):573-84.

http://dx.doi.org/10.1682/JRRD.2012.05.0090

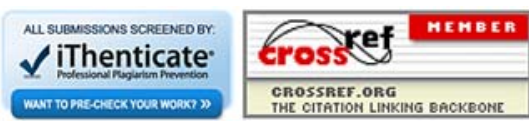

